\title{
Successful treatment of extensively drug-resistant Acinetobacter baumannii ventriculitis with polymyxin $B$ and tigecycline- a case report
}

\author{
Wei Guo, Shao-Chun Guo, Min Li, Li-Hong Li and Yan Qu*
}

\begin{abstract} barrier. drug-resistant Acinetobacter baumannii ventriculitis.

\section{Background}

Acinetobacter baumannii has emerged as a major nosocomial central nervous system infection, with mortality rates ranging from $15 \%$ to $71 \%$ for acinetobacter meningitis [1]. Successful treatments with intraventricular/intrathecal polymyxins have been reported; however, the incidence of potential toxicity was not negligible [2]. Here, we report a successful microbiological cure for extensively drugresistant $A$. baumannii ventriculitis using intravenous and intraventricular polymyxin $B$ together with intravenous tigecycline.
\end{abstract}

Background: Acinetobacter baumannii nosocomial ventriculitis/meningitis, especially those due to drug-resistant strains, has substantially increased over recent years. However, limited therapeutic options exist for the Acinetobacter baumannii ventriculitis/meningitis because of the poor penetration rate of most antibiotics through the blood-brain

Case presentation: A 57-year-old male patient developed ventriculitis from an extensively drug-resistant strain of Acinetobacter baumannii after the decompressive craniectomy for severe traumatic brain injury. The patient was successfully treated with intraventricular and intravenous polymyxin B together with intravenous tigecycline.

Conclusions: The case illustrates intraventricular polymyxin B can be a therapeutic option against extensively

Keywords: Acinetobacter Baumannii, Multidrug resistance, Polymyxin B, Ventriculitis, Intraventricular therapy

\section{Case presentation}

A 57-year-old male patient was transferred from another hospital to the neurosurgical intensive care unit of the Tangdu hospital. The patient had experienced a falling accident at work and then was diagnosed as having a severe traumatic brain injury. The patient underwent a

\footnotetext{
* Correspondence: Yanqu0123@icloud.com

Department of Neurosurgery, Tangdu Hospital, Fourth Military Medical University, Xi'an, Shaanxi 710038, China
}

(c) The Author(s). 2018 Open Access This article is distributed under the terms of the Creative Commons Attribution 4.0 International License (http://creativecommons.org/licenses/by/4.0/), which permits unrestricted use, distribution, and reproduction in any medium, provided you give appropriate credit to the original author(s) and the source, provide a link to the Creative Commons license, and indicate if changes were made. The Creative Commons Public Domain Dedication waiver (http://creativecommons.org/publicdomain/zero/1.0/) applies to the data made available in this article, unless otherwise stated. 
and resistant $(\geq 4 \mu \mathrm{g} / \mathrm{ml})$. For tigecycline, the U.S. Food Drug Administration proposed breakpoints are susceptible $(\leq 2 \mu \mathrm{g} / \mathrm{ml})$, intermediate $(4 \mu \mathrm{g} / \mathrm{ml})$ and resistant $(8 \mu \mathrm{g} / \mathrm{ml})$. A chest computed tomography scan showed lung infiltrates, which were suggestive of pneumonia. Sputum sample was cultured on Columbia agar with $5 \%$ sheep blood and MacConkey at $35{ }^{\circ} \mathrm{C}$ in aerobic conditions for $48 \mathrm{~h}$. The same strain of $A$. baumannii was isolated from the sputum (polymyxin susceptibility was not tested). The antimicrobial therapy was changed to intraventricular (IVT) polymyxin B (50,000 U q24h), intravenous polymyxin B (450,000 U q12h), and tigecycline (50 mg q12h). The patient became afebrile 5 days after the polymyxin $\mathrm{B}$ and tigecycline therapy, with negative CSF cultures thereafter. However, in the treatment process, decreased CSF drainage and a contractible right ventricle were observed gradually. Ten days after the right frontal EVD placement, the right EVD was removed, and another EVD was inserted into the left lateral ventricle. Antimicrobial treatment was switched to IVT polymyxin B (25,000 U q12h), intravenous polymyxin B $(475,000 \mathrm{U}$ $\mathrm{q} 12 \mathrm{~h}$ ), and tigecycline (50 mg q12h). A contractible left ventricle was also observed after the IVT polymyxin $\mathrm{B}$ administration. Next, the antimicrobial regimen was changed by stopping the IVT polymyxin B administration and continuing the intravenous polymyxin $\mathrm{B}(500,000 \mathrm{U}$ $\mathrm{q} 12 \mathrm{~h}$ ) and tigecycline (50 $\mathrm{mg} \mathrm{q} 12 \mathrm{~h}$ ) administration for another 14 days until the patient's clinical conditions were stable. On day 31 of the hospitalization, the patient was discharged.

\section{Discussion and conclusion}

Over the years, Acinetobacter baumannii, which is associated with post-neurosurgical meningitis and ventriculitis, has increasingly been regarded as an important nosocomially acquired pathogen $[1,3]$. Statistical data showed that 3.6$11.2 \%$ of post-neurosurgical meningitis cases are caused by A. baumannii $[2,4]$. Antimicrobial-resistant $A$. baumannii are divided into three categories: multidrug-resistant (MDR), extensively drug-resistant (XDR) and pandrug-resistant (PDR). XDR is defined as non-susceptibility to all penicillins and cephalosporins (including inhibitor combinations), fluroquinolones, aminoglycosides, and carbapenems. [5]. In the present case, XDR A. baumannii that was susceptible only to polymyxin and tigecycline was cultured from the cerebral spinal fluid (CSF) and sputum. As a result of the poor blood-brain barrier penetration, intraventricular (IVT) therapy polymyxin $\mathrm{B}$ was used through the external ventricular derivation (EVD).

Emerging evidence has indicated that intrathecal (IT) or IVT colistin administration is a safe and effective treatment for XDR A. baumannii meningitis [6]. Karaiskos et al. [2]. summarized 36 studies and a total of 81 patients who were diagnosed with meningitis secondary to neurosurgical procedures. A total of $89 \%(72 / 81)$ of the cases treated with IVT/ITH colistin were eventually cured, and the median time to achieve sterilization of the CSF was 4 days. De Bonis et al. [3]. compared the outcomes of the XDR A. baumannii ventriculomeningitis patients treated with intravenous (IV) colistin or IV plus IVT colistin. Compared with $33.3 \%$ of the cases in the IV alone group, $100 \%$ of the cases achieved CSF sterilization (a negative CSF culture result) in the IV + IVT group. The results showed that IVT colistin administration is significantly more effective than IV colistin alone [3]. To the best of our knowledge, polymyxin B and colistin (polymyxin E) were regarded as equivalent because of their similar chemical structures and their activity spectra [7]. However, compared to colistin, there is limited clinical data for the use of polymyxin B in ventriculitis treatment. Piparsania et al [8]. showed successful treatment of multidrug-resistant A. baumannii neonatal meningo-ventriculitis with IVT polymyxin $B$ in combination with IV netilmicin and polymyxin B. IVT polymyxin B (40,000 units per dose) was given alternate day for four weeks. In the present case, sterilization of CSF was detected 5 days after the IVT polymyxin B administration. Hence, we speculate that IVT polymyxin B administration could be as effective as IVT colistin administration.

In the present case, tigecycline was used intravenously. Tigecycline, which belongs to a new class of antibiotics known as the glycylcyclines [9], has demonstrated excellent activity against Acinetobacter strains [10]. Pallotto et al. [11] showed the weak penetration of tigecycline to the CSF in a patient with ventriculo-atrial shunt infection. The average CSF concentration of tigecycline equals to $7.9 \%$ of the serum concentration. That is the reason why tigecycline is not currently recommended for Acinetobacter ventriculitis. Recently, Lauretti et al. reported a successful case of the IVT tigecycline use to treat PDR A. baumannii meningitis [12].

Potential toxicity is a concern associated with local administration of polymyxins. Chemical ventriculitis and meningitis which usually cause fever and altered mental state, are the most severe adverse effects reported with IVT/ITH polymyxin treatment. Karaiskos reviewed the literature and found that out of 81 patients with IVT/ITH colistin administration, chemical meningitis and chemical ventriculitis were diagnosed in $3(3.7 \%)$ and $2(2.4 \%)$ cases, respectively [2]. A recent retrospective study showed that out of 9 patients with IVT colistin administration, no cases of chemical meningitis were encountered [3]. However, to the best of our knowledge, limited reports on the adverse effects of IVT/ITH polymyxin B were published.

In the treatment process, two observations were noted. First, the transient ventricular adhesion due to the IVT polymyxin B administration was observed from the CT scan (Fig. 1). Initially, the IVT polymyxin B was 

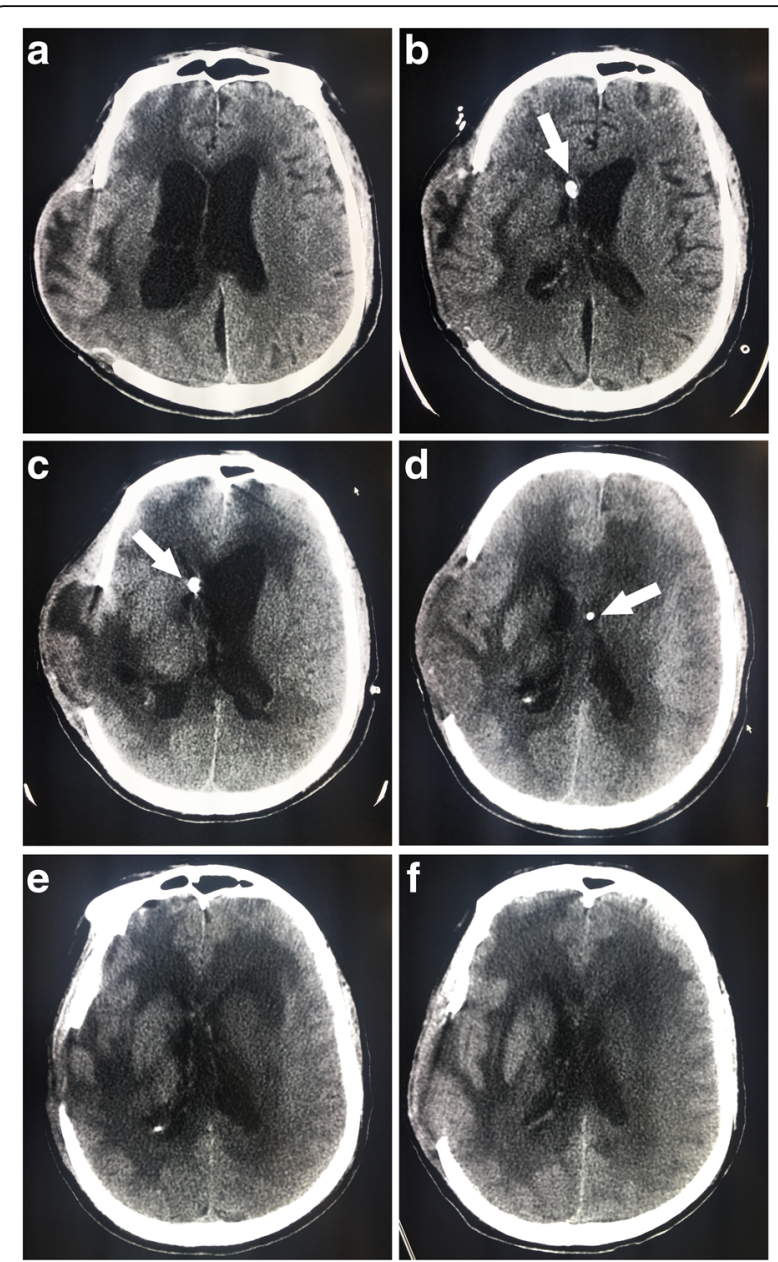

Fig. 1 Transient ventricular adhesion due to intraventricular (IVT) polymyxin B administration observed from CT scan. a CT scan obtained on day 1 of the hospitalization, showing bilateral hydrocephalus. b On day 6 of the hospitalization, CT scan showing the contractible right ventricle and enlarged left ventricle after IVT polymyxin B administration through right EVD (arrows). c On day 11 of the hospitalization, СT scan showing the contractible right ventricle and enlarged left ventricle after IVT polymyxin B administration through right EVD (arrows). d On day 18 of the hospitalization, CT scan showing the contractible left ventricle after IVT polymyxin B administration through left EVD (arrows). e CT scan obtained on day 20 of the hospitalization. $\mathbf{f}$ CT scan obtained on day 23 of the hospitalization

administered through the right EVD. However, decreased CSF drainage and a contractible right ventricle were detected after the IVT polymyxin B administration. Six days later, when the left EVD were used for the polymyxin B administration, left ventricle shrinkage was observed. These phenomena indicate that there is a direct correlation between polymyxin $B$ usage and ventricular adhesion. Second, at the 6-month follow-up, the neurological conditions of the patients had not improved, and the patient was still in a comatose status.
Therefore, we conclude that IVT and intravenous polymyxin B combined with intravenous tigecycline could be an effective therapeutic option in the treatment of XDR A. baumannii ventriculitis. Currently, IVT administration of antibiotics with favorable outcomes is widely reported. However, multicenter randomized studies are still needed to demonstrate the efficacy and safety of intraventricular administration on these patients.

\section{Abbreviations}

CSF: Cerebrospinal fluid; EVD: External ventricular drain; IT: Intrathecal; IV: Intravenous; IVT: Intraventricular; MDR: Multidrug-resistant; PDR: Pandrug-resistant; XDR: Extensively drug-resistant

\section{Acknowledgements}

Not applicable

\section{Funding}

Not applicable

\section{Availability of data and materials}

Data sharing not applicable to this article as no datasets were generated or analysed during the current study.

\section{Authors' contributions}

YQ was in charge of case reviewing and preparation of the manuscript. WG and LHL collected clinic opinions regarding on this case and drafted the manuscript. SCG and ML participated in its coordination and revised the manuscript. All authors read and approved the final manuscript.

\section{Ethics approval and consent to participate}

The study was approved by the Ethics Committee of Tangdu Hospital, Shaanxi, China.

\section{Consent for publication}

Written informed consent was obtained from the next of kin of the patient for publication of this Case report and accompanying images. A copy of the written consent is available for review by the Editor-in-Chief of this journal.

\section{Competing interests}

The authors declared no potential conflicts of interest with respect to the research, authorship, and/or publication of this article.

\section{Publisher's Note}

Springer Nature remains neutral with regard to jurisdictional claims in published maps and institutional affiliations.

Received: 6 June 2017 Accepted: 7 February 2018

Published online: 14 February 2018

\section{References}

1. Kim BN, Peleg AY, Lodise TP, Lipman J, Li J, Nation R, Paterson DL. Management of meningitis due to antibiotic-resistant Acinetobacter species. Lancet Infect Dis. 2009;9(4):245-55.

2. Karaiskos I, Galani L, Baziaka F, Giamarellou H. Intraventricular and intrathecal colistin as the last therapeutic resort for the treatment of multidrug-resistant and extensively drug-resistant Acinetobacter baumannii ventriculitis and meningitis: a literature review. Int J Antimicrob Agents. 41(6):499-508.

3. De Bonis P, Lofrese G, Scoppettuolo G, Spanu T, Cultrera R, Labonia M, Cavallo MA, Mangiola A, Anile C, Pompucci A. Intraventricular versus intravenous colistin for the treatment of extensively drug resistant Acinetobacter Baumannii meningitis. Eur J Neurol. 23(1):68-75.

4. Wang KW, Chang WN, Huang CR, Tsai NW, Tsui HW, Wang HC, Su TM, Rau CS, Cheng BC, Chang CS. Post-neurosurgical nosocomial bacterial meningitis in adults: microbiology, clinical features, and outcomes. J Clin Neurosci. 2005;12(6):647-50.

5. Magiorakos AP, Srinivasan A, Carey RB, Carmeli Y, Falagas ME, Giske CG, Harbarth S, Hindler JF, Kahlmeter G, Olsson-Liljequist B. Multidrug-resistant, 
extensively drug-resistant and pandrug-resistant bacteria: an international expert proposal for interim standard definitions for acquired resistance. Clin Microbiol Infect. 18(3):268-81.

6. Hoenigl M, Drescher M, Feierl G, Valentin T, Zarfel G, Seeber K, Krause R, Grisold A. Successful management of nosocomial ventriculitis and meningitis caused by extensively drug-resistant Acinetobacter baumannii in Austria. Can J Infect Dis Med Microbiol. 24(3):e88-90.

7. Cai Y, Lee W, Kwa AL. Polymyxin B versus colistin: an update. Expert Rev Anti-Infect Ther. 13(12):1481-97.

8. Piparsania S, Rajput N, Bhatambare G. Intraventricular polymyxin B for the treatment of neonatal meningo-ventriculitis caused by multi-resistant Acinetobacter Baumannii- case report and review of literature. Turk J Pediatr. 2012;54:548-54.

9. Pankey GA. Tigecycline. J Antimicrob Chemother. 2005;56(3):470-80.

10. Karageorgopoulos DE, Falagas ME. Current control and treatment of multidrug-resistant Acinetobacter Baumannii infections. Lancet Infect Dis. 2008:8(12):751-62

11. Pallotto C, Fiorio M, D'Avolio A, Sgrelli A, Baldelli F, Di Perri G, De Socio GV. Cerebrospinal fluid penetration of tigecycline. Scand J Infect Dis. 46(1):69-72

12. Lauretti L, D'Alessandris QG, Fantoni M, D'Inzeo T, Fernandez E, Pallini R, Scoppettuolo G. First reported case of intraventricular tigecycline for meningitis from extremely drug-resistant Acinetobacter Baumannii. J Neurosurg. 2016;19:1-4.

\section{Submit your next manuscript to BioMed Central and we will help you at every step:}

- We accept pre-submission inquiries

- Our selector tool helps you to find the most relevant journal

- We provide round the clock customer support

- Convenient online submission

- Thorough peer review

- Inclusion in PubMed and all major indexing services

- Maximum visibility for your research

Submit your manuscript at www.biomedcentral.com/submit 